Chirurg 2020 $91: 432-434$

https://doi.org/10.1007/s00104-020-01182-y

Online publiziert: 15. April 2020

(c) Springer Medizin Verlag $\mathrm{GmbH}$, ein Teil von Springer Nature 2020

\author{
Joachim Jähne ${ }^{1,2}$ \\ ${ }^{1}$ Deutsche Gesellschaft für Chirurgie e. V., Berlin, Deutschland \\ ${ }^{2}$ Klinik für Allgemein- und Viszeralchirurgie, Diakovere Friederikenstift und Henriettenstift, Hannover \\ Deutschland
}

\title{
Die Corona-Krise und ihre Auswirkungen auf die Chirurgie
}

belegt werden können. Zudem drohen Versorgungsengpässe bei Beatmungsschläuchen und Medikamenten wie z.B. Propofol, so dass auch aus diesen Gründen eine deutliche Einschränkung der operativen Tätigkeit resultiert. Es steht durchaus zu befürchten, dass auf dem Gebiet der Viszeralchirurgie gerade onkologische Patienten aufgrund fehlender Ressourcen die erforderlichen Eingriffe nicht mit der gebotenen Dringlichkeit erhalten können. Die dadurch bedingten Absagen und Verschiebungen von (vermeintlich) nicht dringlichen Eingriffen lösen bei den betroffenen Patienten Sorgen über die weitere Behandlung aus. Welcher Patient als dringlich einzuplanen ist und welcher verschoben werden muss, obliegt einzig der ärztlichen Entscheidung des verantwortlichen Chirurgen und kann nicht aufgrund allgemeiner Empfehlungen entschieden werden. Es gilt allerdings zu verhindern, dass chirurgische Patienten wegen Covid-19 versterben, ohne daran erkrankt zu sein.

Mit hohem logistischem Aufwand haben die Krankenhäuser die planbaren Eingriffe durch telefonische Kontaktaufnahme mit den Patienten abgesagt, ohne einen neuen Operationstermin nennen zu können. Sämtliche Sprechstundenaktivitäten wurden überwiegend komplett heruntergefahren. Damit tritt für alle chirurgischen Kliniken in Deutschland perspektivisch ein erheblicher Stau an später durchzuführenden Operationen auf, so dass bereits jetzt klar ist, dass zukünftig bei begrenzter Infrastruktur und eingeschränkten Personalressourcen eine Priorisierung der chirurgischen Leistungen wahrscheinlich notwendig ist. Durch die Absage der Sprechstunden wurde aber auch deutlich, dass bis dato noch für unmöglich gehaltene telefonische oder digitale Beratungen nach dem erst kürzlich aufgehobenen Verbot der Fernbehandlung durchaus zur Regel geworden sind. Es ist $\mathrm{zu}$ hoffen, dass diese „Modernisierung“ der Sprechstundentätigkeit auch über die Corona-Krise hinaus Bestand haben wird.

Auffällig ist in den letzten zwei bis drei Wochen nach eigener Erfahrung und auch nach zahlreichen Berichten von Notfallmedizinern die Anzahl an Herzinfarkten und Schlaganfällen sowie die Notfalleingriffe deutlich rückläufig sind. In der Viszeralchirurgie sind in den letzten Tagen praktisch keine akuten Gallenblasenentzündungen, keine Blinddarmentzündungen und auch keine Divertikulitiserkrankungen notfallmäßig stationär aufgenommen worden. Der Anteil der Frakturen ist ebenfalls in einem bis vor kurzem noch nicht für möglich gehaltenen Umfang $\mathrm{zu}$ rückgegangen. Die Ursachen für diese Rückgänge sind vielschichtig und haben u. a. mit der Einschränkung der Bewegungsfreiheit, einer größeren Häuslichkeit mit mehr Ruhe sowie der Angst vor einer Covid-19-Infektion im Krankenhaus zu tun. Die elektiven und NotfallFallzahlrückgänge werden sich in den nächsten Wochen bei einem noch nicht absehbaren Ende der Corona-Pandemie weiter verstärken, und es muss allen Beteiligten klar sein, dass dies erhebliche Auswirkungen auf die Weiterbildung in unserem Fachgebiet hat. Es ist davon auszugehen, dass sich bei vorsichtiger jetziger Schätzung die Weiterbildungszeit aller Voraussicht nach um mindestens 
sechs, wenn nicht 12 Monate verlängern wird. In diesem Zusammenhang ist $\mathrm{zu}$ fordern, dass im Interesse der Weiterbildung des chirurgischen Nachwuchses die meist befristeten Weiterbildungsverträge unbürokratisch entsprechend verlängert werden. Hinzu kommt in diesem Zusammenhang, dass in vielen Kliniken Chirurginnen und Chirurgen für die Beherrschung der Covid-19-Situation in anderen Bereichen wie der Intensivstation, der Intermediate Care Unit oder im Triage-Zelt zum Einsatz kommen. Und dennoch ist erkennbar, dass die bisher schon knapp bemessenen Personalressourcen im ärztlichen (und pflegerischen) Bereich nicht ausreichen werden, um allen Anforderungen gerecht werden zu können. In Anbetracht der möglichen Auswirkungen einer CoronaInfektion für die betroffenen Patienten werden all diese Veränderungen und Einschränkungen vollumfänglich seitens der Chirurgie mitgetragen, da diese Infektionswelle die Zusammenarbeit aller Ärztinnen und Ärzte erfordert. In diesem Zusammenhang sollten die leitenden Ärzte und Oberärzte dann auch konsequenterweise von der Erfüllung klinikinterner eventueller Zielvereinbarungen, die sich selbstverständlich nicht auf Fallzahlsteigerungen beziehen sollten, entlastet werden. Darüber hinaus wird empfohlen, eventuelle Audits für (Re-)Zertifizierungen z.B. seitens der Deutschen Krebsgesellschaft oder der DGAV für das Jahr 2020 auszusetzen und bestehende Zertifizierungen um ein Jahr zu verlängern. Auch dies wäre ein Zeichen der Solidarität und würde die Kliniken substantiell entlasten.

Aus Sicht der chirurgischen Kliniken ist es allerdings beunruhigend, dass die von der Politik vorgeschlagenen Regelungen des Covid-19-Krankenhausentlastungsgesetzes zum Ausgleich der Erlösrückgänge sicher nicht ausreichen werden. Die tagesbezogene Ausgleichszahlung von $560 €$ pro Bett, der fallbezogenen Zuschlag von $50 €$ pro Schutzausrüstung (an der neben den Praxen und Pflegeeinrichtungen auch in den Kliniken noch z.T. gravierender Mangel herrscht) und die Erhöhung des Pflegeentgeltwertes um etwa $38 €$ auf $185 €$ werden kaum ausreichen, um die Li- quidität der Krankenhäuser zu erhalten. Dies verdeutlicht folgendes Beispiel: Für eine einfache Thyreoidektomie mit einem dreitägigen stationären Aufenthalt (inclusive OP-Tag) sieht das DRG-System einen Erlös von rund $3700 €$ vor. Die vorgesehene tagesbezogene Ausgleichszahlung für diesen nicht durchgeführten Eingriff liegt bei knapp $1700 €$. Man muss nicht Mathematik oder Betriebswirtschaft studiert haben, um zu erkennen, dass dies bei Konstanz der Fixkosten nicht ausreichend ist. Auch die zusätzliche Bonuszahlung von 50.000€ pro zusätzlich geschaffenem Intensivbett ist nicht dazu angelegt, Liquiditätsengpässe zu vermeiden. Natürlich kann erst nach dem Ende der Krise überprüft werden, ob die vorgesehenen Ausgleichszahlungen auskömmlich waren, um Defizite bei den Krankenhäusern zu verhindern. Ohne in weitere Detailberechnungen einzusteigen, ist jedoch bereits jetzt $\mathrm{zu}$ konstatieren, dass möglicherweise kleinere Kliniken mit einer bis dato geringen Infrastruktur von den CoronaAusgleichszahlungen profitieren werden, während Universitätskliniken, Maximalversorger und auch Fachkliniken, die üblicherweise eine sehr große und differenzierte Infra- und Personalstruktur vorhalten, in erhebliche Liquiditätsengpässe geraten. Es ist sehr zu hoffen, dass in der Kürze der Zeit, in der sich die Kliniken auf die Corona-Krise eingestellt haben, auch von politischer Seite über die öffentlich vorgetragenen Bekenntnisse zu unserem Gesundheitssystem hinaus ein Signal erfolgt, dass in dieser schwierigen Situation kein Krankenhaus der Bundesrepublik Deutschland vor Liquiditätsschwierigkeiten gestellt wird. Denn sonst ist es sehr leicht möglich, dass diejenigen, die jetzt beklatscht werden, in etwa sechs bis acht Wochen kein Gehalt mehr erhalten. Die Auswirkungen auf unser Gesundheitssystem wären vernichtend ...

\section{Korrespondenzadresse}

Prof. Dr. med. Joachim Jähne, FACS, MBA

Klinik für Allgemein- und Viszeralchirurgie, Diakovere Friederikenstift und Henriettenstift Marienstraße 72-90, 30171 Hannover,

Deutschland

joachim.jaehne@diakovere.de

\section{Einhaltung ethischer Richtlinien}

Interessenkonflikt. J. Jähne gibt an, dass kein Interessenkonflikt besteht.

Für diesen Beitrag wurden vom Autor keine Studien an Menschen oder Tieren durchgeführt. Für die aufgeführten Studien gelten die jeweils dort angegebenen ethischen Richtlinien. 
Hier steht eine Anzeige.

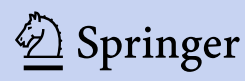

